
Codiseño
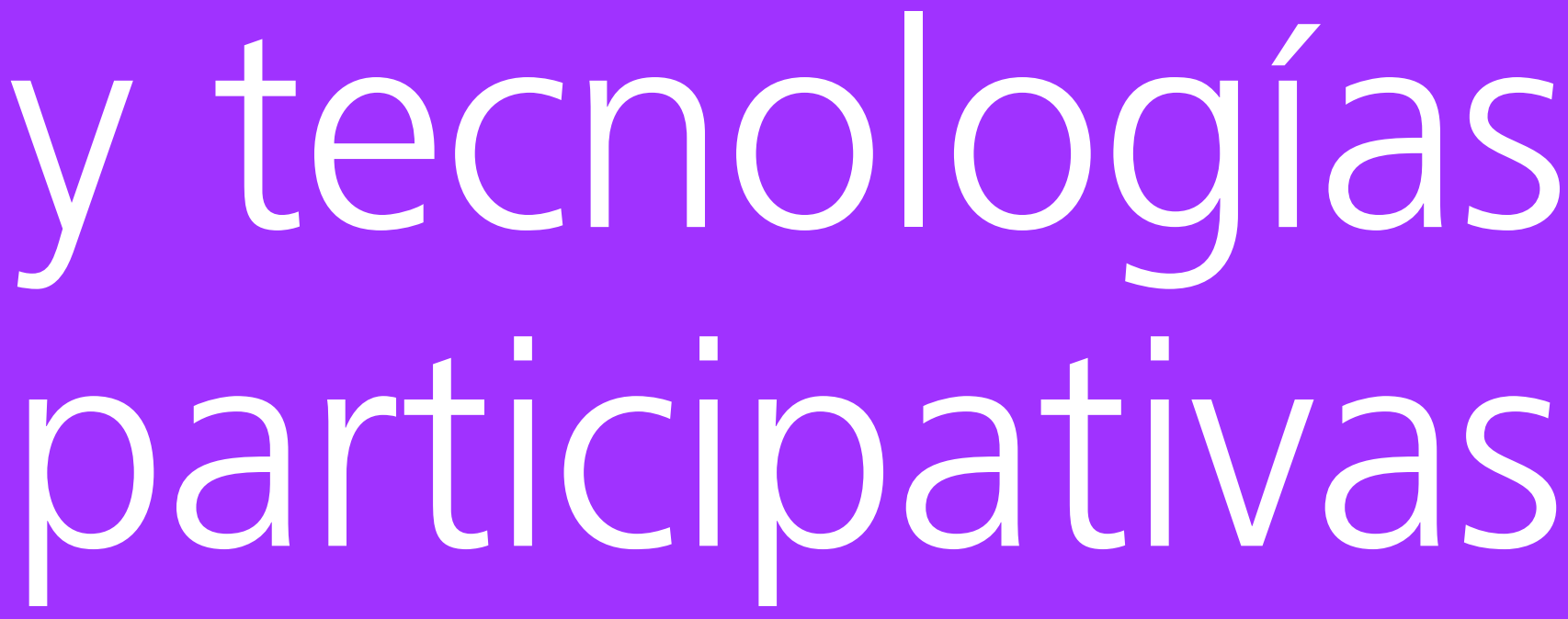

Diego Alatorre

Alatorre, D. (2015). Codiseño y tecnologías participativas, Economía Creativa. (04) otoño-invierno 2015, México: pp. 8-29. 


\section{Codiseño y tecnologías participativas}

MDI. Diego Alatorre ${ }^{1}$

\section{Resumen}

Propósito | El objetivo del presente estudio es suprimir identificar los factores que pueden ofrecer a los estudiantes de diseño, las capacidades necesarias para desarrollarse plena y responsablemente en el contexto laboral, uno en el que la opción de trabajar para alguien más, no sea más su objetivo, sino la creación de proyectos personales, participativos y en búsqueda del bien común.

Método | Los resultados aquí expuestos se sustentan en el trabajo y la investigación que he realizado durante los últimos dos años impartiendo la materia de CoDiseño - Diseño Colaborativo en el CIDI+UNAM y recientemente en la materia de Innovación y Modelos de Negocio en el ITAM. Aunado a mi experiencia como docente, presento la investigación que sustenta mi práctica educativa y que a su vez permea los objetivos, el diseño y la implementación de los curso que facilito.

Parte sustancial de mi quehacer consiste enseñar a los estudiantes a usar y adaptar herramientas que les faciliten la Investigación Basada en la Comunidad, el Análisis Cualitativo Consensuado y la Elaboración Colectiva de Ideas. De esta manera, los alumnos, aprenden a construir sondas, prototipos y otros artefactos para ejercer el proceso de diseño, desde una perspectiva participativa.

Resultados principales | Se exponen seis consideraciones estratégicas que estructuran un marco teórico y metodológico para el desarrollo de proyectos educativos que busquen la creación de nuevas comunidades o el fortalecimiento de comunidades previamente existentes: (1) el diseño de planes de estudios flexibles y permeables a las necesidades y aspiraciones de los estudiantes, (2) el cambio de enfoque que implica diseñar procesos e interacciones en vez de producto materiales, (3) la procuración de nuevos y mejores satisfactores, (4) la identificación de la producción a escala humana como principio de la creación de bienes y servicios regenerativos que satisfagan las necesidades de los múltiples

\footnotetext{
1 diego.alatorre@cidi.unam.mx, Universidad Nacional Autónoma de México.
} 
involucrados en el proyecto, (5) la integración de dinámicas participativas que vinculen a los estudiantes con personas ajenas a la universidad y (6) el diseño de herramientas mentales, materiales, digitales y espaciales que propicien el intercambio de experiencias y conocimientos.

Palabras clave | Diseño, educación, participación, comunidad.

\begin{abstract}
Objective | The purpose of this paper is to identify the tools to offer design students the right abilities to develop themselves broadly and responsible in a new professional context, one in which the option to work for someone else is no longer their objective, but the creation of intimate and participatory projects in the scope for a common good.

Method | The results exposed here are based on research and work I have undertaken over the last two years facilitation the course of CoDiseño at CIDI+UNAM and recently the course of Innovation and Model Business Design at ITAM. Aligned to my experience as teacher, I present here the research that structures my educational practices and permeates the objectives, procedures and implementation of the course I teach.

A substantial part of my practice consists on teaching students to utilize and redesign different tools to facilitate Community Based Research, Consensual Qualitative Analysis and Collaborative Idea Generation. This way, students learn to build probes, prototypes and other artefacts to exert the design process within a participatory approach.

Main results | During the text, I highlight the characterization of six strategic consideration to structure a methodological and theoretical framework aimed to develop educational projects that foster the creation of new communities or the strengthening of existing ones: (1) the design of flexible curricula that allows for the integration of students needs and aspirations, (2) the shift on the design scope from product to process, (3) the identification of the human scale production as the principle of creation of regenerative goods and services to satisfy multiple stakeholders' needs, (4) the integration of participatory dynamics to link students to external parties and (5) the design of mental, material, digital and spatial tools that promote the exchange of experiences and knowledge.
\end{abstract}

Keywords | Design, education, participation, community. 


\section{Introducción}

Humberto Maturana, biólogo, filósofo y pedagogo chileno escribió en Metadesign (1997) que "nosotros seres humanos podemos hacer lo que imaginemos siempre y cuando respetemos las coherencias estructurales del dominio en el que operamos". Él mismo advierte del terror que esta idea puede llegar a sugerir, "pero -continúa Maturana- no tenemos que hacer todo lo que imaginemos, podemos escoger, y es entonces cuando nuestro comportamiento como seres humanos socialmente responsables importa".

Estando limitados tanto por nuestra creatividad como por el mismo entendimiento de las características del sistema en donde operamos, el reto al que nos enfrentamos actualmente se debe abordar desde ambos flancos y en paralelo, desarrollando las capacidades creativas que nos permitan entrever nuevos horizontes y generando más y mejor conocimiento, que nos permita identificar las especificidades del contexto donde pretendamos aplicar nuestros esfuerzos.

Maturana escribe también que aunque "la noción de realidad está cambiando, no así nuestra vida en relación a ella". Es así como actualmente, aunque mucho se habla de innovación, en gran medida, seguimos haciendo y pensando las cosas de maneras muy similares a nuestros antepasados: seguimos las mismas reglas que nosotros no inventamos e incluso interactuamos con valores que ya no promovemos.

¿Cómo, entonces, pretendemos innovar dentro de un sistema en el que se tiene aversión al cambio, donde las estructuras son rígidas, tanto como las mentes que fueron formadas bajo los ideales de la libre competencia, el individualismo y la apatía política? Más aún, ¿somos conscientes de la tremenda responsabilidad que tenemos al asumirnos como personas proactivas, creadoras de realidades propias y ajenas?

La cuestión ya me daba vértigo cuando empecé mi vida laboral diseñando muebles, pensando en la cantidad de personas que podrían lastimarse por un tornillo mal puesto o un ensamble mal calculado; sin embargo como docente, observando la facilidad con la que algunas ideas pueden "plantarse" y lo fértiles que son las cabezas de los más jóvenes, creo que la pregunta ha tomado un tono bastante más sensible. 
Aunque parezca trivial, sabemos que la simple perspectiva con la que uno encara cualquier asunto, puede cambiar determinantemente el rumbo de los acontecimientos. Con cada acción que hacemos, forjamos activamente nuestro presente y el de los que nos rodean. Cada cosa que hacemos importa y tiene repercusiones directas e indirectas en todo lo que nos rodea.

La pregunta, entonces, va mucho más allá de definir las consideraciones temáticas que deben ser tratadas dentro o fuera del salón de clases; implica identificar los valores que se sustentan a través del contenido y se ponen en práctica en el trato diario entre profesores y estudiantes, entre clientes y proveedores de servicios y en general entre todo grupo de personas vinculadas en torno a un proyecto colectivo.

Es así que entre las muchas instancias educativas que se encuentran en etapa de crisis, el gremio de diseño industrial se encuentra especialmente tambaleante frente al desmoronamiento de los cimientos sobre los que originalmente fue erguido.

Como gremio, sociedad e individuos, la mayoría de nosotros nacimos bajo la influencia del capitalismo. Progresar significaba construir las ciudades más grandes, erigir los edificios más altos y manejar los coches más rápidos; se nos enseñó a acumular recursos por miedo a la escasez, a no copiar en los exámenes, a no contradecir a los mayores y a saludar a una bandera que normaliza el nepotismo.

Distinto es el caso de algunos jóvenes que hoy ocupan los salones de clases, quienes (aquejados por otros fantasmas sociales, de los cuales no voy a elaborar en el presente documento) han crecido en un ambiente presidido por el intercambio, en donde la información es abundante y se permiten copiar y pegar para crear nuevas canciones, donde se hacen una y varias cosas al mismo tiempo y donde importa más vivir experiencia significativas que la posesión de bienes materiales.

El cambio hacia una sociedad en donde se valore a la comunidad por sobre el individuo y dentro de la cual se respete el medio ambiente antes que las ganancias monetarias, no puede venir si actuamos bajo los mismos valores de industrialización y consumismo que definieron el rumbo del diseño industrial en el siglo pasado. Como dice Paul 
Manson (2015) en su reciente artículo El comienzo del fin del capitalismo ha llegado; es tiempo ya de cambiar nuestro paradigma y para eso, debemos comenzar por creernos capaces de hacerlo.

\section{CoDiseño o ¿cómo diseñar para una sociedad más equitativa?}

No pretendo aparentar que conozco la receta o incluso que ésta exista, sin embargo, después de experimentar activa y reflexivamente a través del diseño y la implementación del curso taller de CoDiseño, he identificado ciertas cualidades interactivas que dan sentido y razón a una nueva manera de pensar, ejercer y reproducir el proceso de diseño de manera inclusiva y colectiva.

CoDiseño se impartió en el CIDI+UNAM por primera vez en el primer semestre de 2014 y desde entonces se ha repetido tres veces, y más de 50 alumnos han cursado la clase. Los objetivos de la materia son:

1 Ofrecer un marco teórico para la conceptualización y el desarrollo de proyectos multidisciplinarios.

2 Practicar, diseñar y construir herramientas que faciliten el desempeño de múltiples participantes dentro de procesos colaborativos.

3 Vincular a estudiantes y profesionales mediante el desarrollo de dinámicas participativas con empresas sociales, privadas y públicas.

Durante el curso, los alumnos aprenden y ponen en práctica técnicas de liderazgo (Acaroglu, 2015) y facilitación creativa (Tassoul, 2009) que le permiten obtener información estratégica para el desarrollo de un concepto de diseño plural y coherente, acorde a al contexto estudiado. 


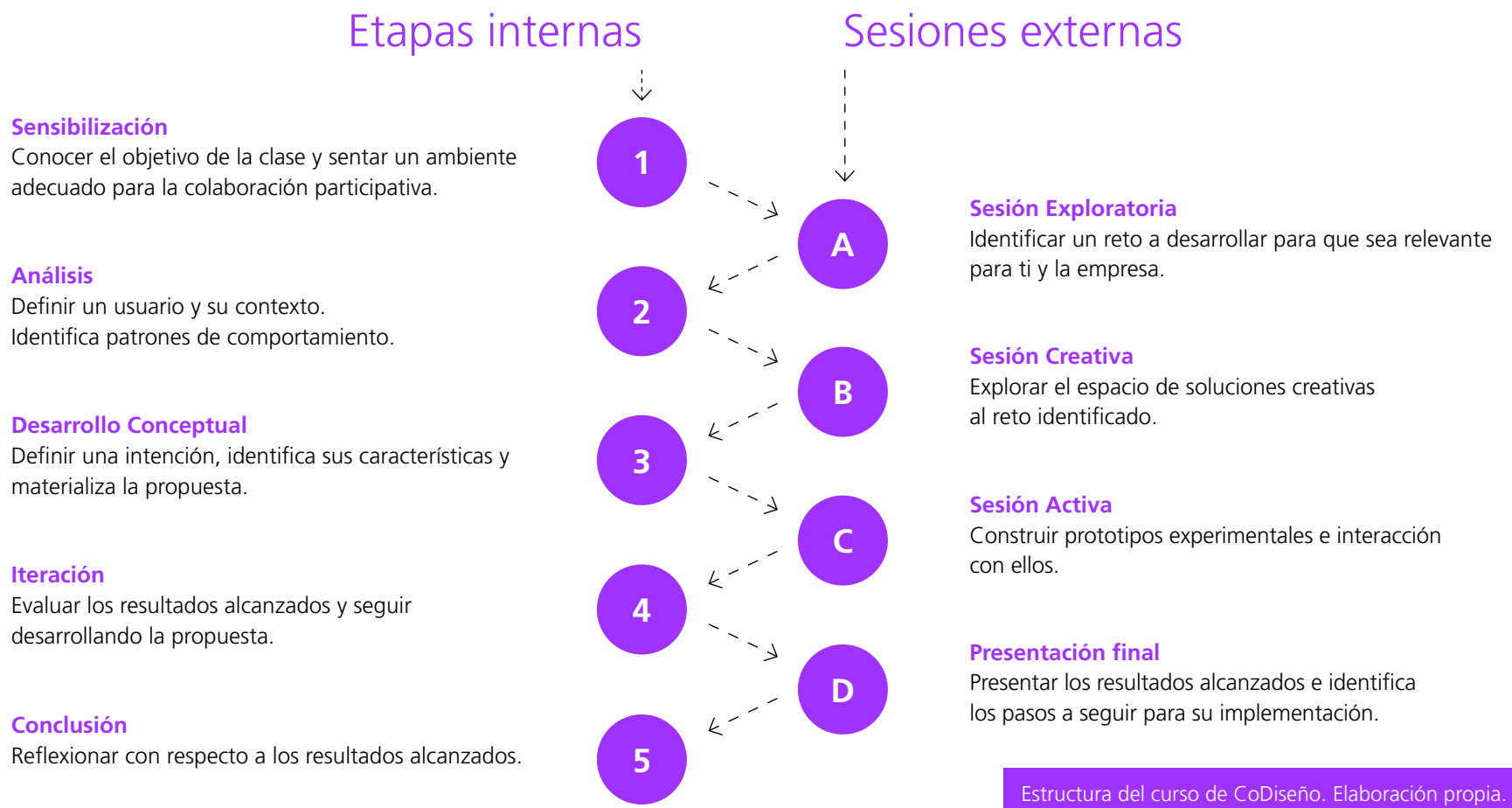

El caso particular que aquí detallo, hace referencia a la experiencia llevada a cabo durante el semestre académico transcurrido entre enero y mayo de 2015, en donde 15 alumnos del CIDI colaboraron con miembros del colectivo de diseño, arquitectura y oficios WeAreTodos (Ciudad de México).

El curso suele seguir una estructura inspirada en el proceso de diseño descrito por Tim Brown (2008) a lo largo de 16 semanas de clases. Este semestre no fue la excepción, aunque sí se refinó más la seriación de actividades, quedando el calendario dividido en cinco etapas de trabajo, materializadas en sesiones internas teórico-prácticas en donde se estudiaron los contenidos temáticos dentro del salón y que fueron procedidas por cuatro sesiones externas, dentro de las instalaciones de WeAreTodos (WAT), a través de las cuales se aplicaron las teorías y las herramientas en proyectos reales. Por lo general, la mayor parte de las actividades se llevaron a cabo en equipo, con excepción del reporte de lectura que se encargó al principio del semestre (Maturana, 1997 y Sanders \& Stappers, 2008) y el plan de trabajo de una sesión externa, en donde cada uno de los estudiantes participó como facilitador, estando a cargo de definir un objetivo para la sesión, facilitar las actividades a realizar y documentar el proceso. 


\section{Sensibilización}

Las primeras clases comenzamos por establecer el espacio de trabajo dentro del salón de clase como uno de creación colaborativa y voluntaria, dentro del cual, cada uno de los involucrados en el proyecto respeta el punto de vista de los otros y juntos aportamos lo mejor de nosotros. Así pues, la primera tarea que les pedí, fue un contrato de aprendizaje, donde cada uno de los alumnos se compromete a aprender al menos tres cosas y las presentan en técnica libre. Los resultados suelen ser muy divertidos y conmovedores. Este semestre, la participación mejor lograda fue la de una alumna que escribió una canción al y la cantó delante de todo el grupo.

La segunda clase se crean equipos y se procura que cada equipo asuma una identidad colectiva a través de un collage participativo y para la tercera semana, se presentan los casos de trabajo. En este caso Luis López y Carlos Zimbrón, dos de los fundadores del WAT se presentaron ante los alumnos y con ellos, presentaron también los cuatro casos a los que los estudiantes se abocarían durante el semestre. Los retos puestos sobre la mesa fueron los siguientes:

- Una instalación interactiva que comunique lo que comparten todos los socios de la comunidad WeAreTodos (WAT).

- Un Laboratorio culinario que conjugue ciencia, arte y comida dentro de un espacio colaborativo de creación y recreación gastronómica.

- Un artículo conmemorativo que personifique y comunique la historia, filosofía y hermenéutica de las FuckUpNights (FUN).

- Un asistente organizativo para ayudar a jóvenes diseñadores a gestionar las labores administrativas, creativas y financieras que sus emprendimientos les demandan.

A través de un proceso de votación abierta, los estudiantes hicieron grupos y se dividieron los cuatro retos, de manera que formaron tres equipos de cuatro integrantes (I, II y III) y uno de tres (IV). 


\section{A. Sesión exploratoria}

Durante cada sesión externa, se lleva a cabo un esquema de trabajo similar: un alumno se encarga de facilitar la sesión en la búsqueda de un objetivo planteado en equipo durante la sesión previa. Los alumnos se presentan en las instalaciones de la empresa y desarrollan en equipo una serie de actividades escogidas por el facilitador de la sesión.

En la sesión externa los alumnos, junto con los representantes de las cuatro empresas, investigaron el contexto (Sanders et al, 2012) y las relaciones entre los múltiples involucrados desde una perspectiva de investigación basada en la comunidad (Schlierf et al, 2008) con el fin de identificar los factores determinantes a tomar en cuenta en los diferentes casos de estudio.

Al final de la sesión los alumnos deberán contar con una interpretación grupal de la tarea a realizar durante el semestre. De acuerdo con Olena Klimenko (2008), el desarrollo pleno de las capacidades creativas de los estudiantes, se lleva a cabo en el momento en que les permitimos tomar un rol activo dentro del proceso educativo y no los asumimos "como organismos que reaccionan pasivamente al estímulo, subordinándose a la lógica del reforzamiento". Así pues, trabajando en equipo, cada grupo reformuló en términos propios, el reto a atacar. Los objetivos acordados se plasmaron dentro de un acuerdo de colaboración y firmado entre ambas partes: los estudiantes y los representantes de las empresas.

\section{Análisis}

De vuelta en el salón de clases y durante dos sesiones más, los estudiantes repasaron en equipo la información recopilada durante la sesión exploratoria, al mismo tiempo que identificamos huecos en la investigación. A continuación, estudiamos los procesos mentales mediante los cuales generamos nuevas y mejores ideas (Tassoul, 2009), esta suele ser una de mis clases favoritas. Durante dos semanas ponemos en práctica diferentes técnicas para facilitar la creatividad de uno y de los demás. Mientras más ejercitamos el músculo creativo, mayor seguridad tendremos para facilitar la creatividad de otras personas. A través de las sesiones internas, los estudiantes se preparan para facilitar las sesiones externas. 


\section{B. Sesión creativa}

Durante una nueva sesión externa, otros cuatro alumnos facilitan nuevas dinámicas de trabajo. Empezando por exponer el motivo y el objetivo de la sesión, se desarrollan diferentes actividades en paralelo con el fin de elaborar la mayor cantidad de soluciones posibles al reto identificado durante las sesiones anteriores. Juntos, en equipos, los participantes desarrollan dibujos, mapas mentales y primeros prototipos que les permiten tomar decisiones desde una perspectiva participativa.

Al final del día, cada equipo deberá haber obtenido una línea de trabajo clara alrededor de la cual se desarrollarán diferentes propuestas de diseño.

\section{Desarrollo conceptual}

Cuando los equipos están bien balanceados, con el interés de todos los participantes y dedicados a un proyecto personal, la conceptualización de soluciones de diseño nos permite dejar que las cosas tomen su propia forma en función de entregarnos a la creación de lo innombrable. Es en ese espacio de incertidumbre, donde a través de la prueba y el error, las cosas comienzan a cobrar significado.

A través de diferentes actividades, como juegos de rol, lluvias de ideas y discusiones en equipo, empezamos a construir los primeros prototipos, mismos que materializan una pregunta, una hipótesis. Hacemos unas primeras pruebas dentro del CIDI y nos preparamos para la siguiente sesión externa.

\section{Sesión activa}

Facilitada por otro alumno diferente a los que ya facilitaron las sesiones anteriores, la sesión activa consiste en validar las decisiones de diseño con los representantes de las empresas y posibles usuarios. Durante ésta, los participantes interactuaron con distintos prototipos experimentales diseñados por los alumnos con el objetivo de poner a prueba sus ideas y buscando recibir retroalimentación que les permita mejorar y sus propuestas. 


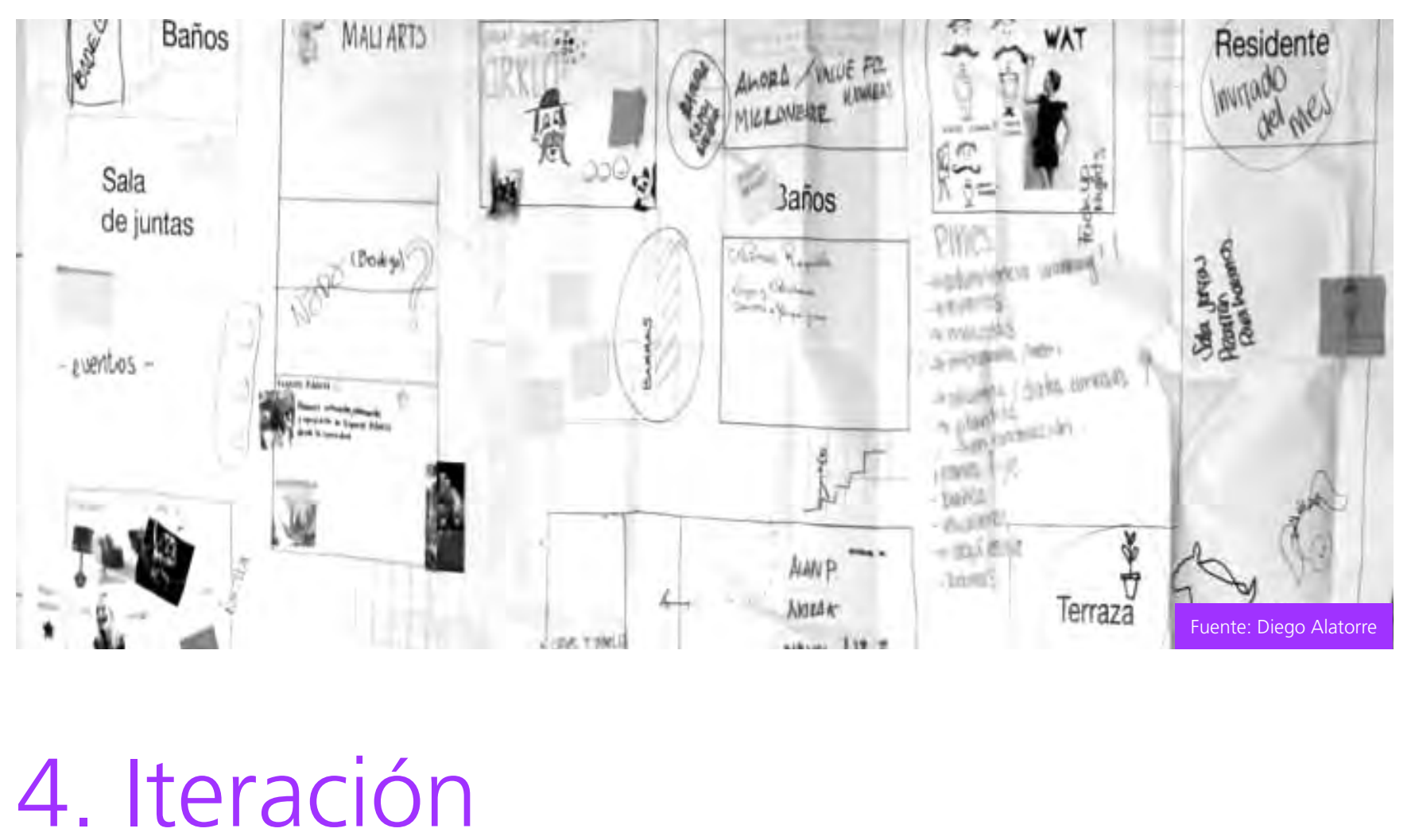

En función de los resultados obtenidos en la sesión activa, los estudiantes, de vuelta al salón de clases, terminan por adecuar sus prototipos y se preparan para la presentación final. Durante las dos últimas semanas, los estudiantes terminan por definir las características finales de la solución que presentarán a los representantes de las empresas para las que trabajan.

Los segundos prototipos son cuestionados, evaluados y refinados con el fin de volverlos a validar y frente a la comunidad WAT en un evento final de clausura. Finalmente, se trabaja en la presentación de los proyectos mediante técnicas de comunicación efectiva.

\section{Presentación final}

La última sesión externa se llevó a cabo, de nuevo en las instalaciones del WAT. A diferencia de las demás, en donde los equipos trabajaron por separado, la Presentación Final se llevó a cabo de manera que cada equipo trajo previamente preparada una actividad para realizarse entre toda la comunidad presente en el evento. A través de las diferentes actividades, los equipos introdujeron a su audiencia a una atmósfera adecuada para la presentación de cada propuesta. De esta manera, comenzó el equipo de alumnos trabajando para el 
proyecto del asistente organizativo con una actividad de calentamiento, donde los participantes tenían que caminar siendo guiados por sus compañeros a las orillas del patio. Al terminar la dinámica, presentaron su propuesta: un dispositivo electrónico con temporizador y alarma que notifica al usuario el tiempo deseado para realizar una actividad y lo motiva a terminar dentro del tiempo.

El siguiente equipo comenzó por hacer un rally alrededor del espacio y en seguida presentaron su instalación interactiva que comunica lo que comparten Todos los socios de la comunidad WAT: un directorio de las empresas construido a partir de un módulos de cerámica con tres zonas interactivas: un área para comunicar el espíritu de la empresa, un espacio para dejar recados a los otros miembros de la comunidad y un contenedor para sembrar una planta-insignia. Un segundo módulo contiene la misma planta-insignia y se cuelga al lado de cada oficina.

El tercer equipo proyectó un video sobre gente fracasando y facilitó un juego en donde, por equipos, los jugadores debían adivinar lo que uno actuaba. A continuación presentaron un artículo promocional y conmemorativo para FuckUpNights: un kit de emergencia que guía al usuario a través de las diferentes etapas que requiere el duelo de vivir un fracaso.

La sesión culminó con la presentación del Laboratorio culinario en donde los asistentes preparamos diferentes recetas, guiados por la intuición y documentando el proceso con el fin de enmarcar una experiencia colectiva y replicable, donde se premiara la colaboración y la experimentación. Terminamos, de manera dinámica y divertida, compartiendo los distintos platos preparados en parejas.

\section{Reflexión}

Al final de cada sesión externa, suelo pedir un reporte final en donde cada alumno reflexiona acerca de cada una de las dinámicas realizadas durante el semestre. Junto con el reporte, les pido, tanto a los estudiantes, como a los representantes de las empresas con 
las que colaboramos, que respondan un cuestionario con retroalimentación. En general, los comentarios suelen ser positivos y en caso de que no, hablo con el equipo en cuestión para identificar las posibles causas y soluciones.

Una de las respuestas más agradables de leer, fue la de Andrea, una alumna que dice que "Esta clase es muy diferente a la mayoría de las clases del CIDI lo cual a mí me parece excelente porque te hace ver el diseño desde otra perspectiva. Creo que la manera en que se trabaja en clase es la manera en que deberían de ser siempre porque te obliga a pensar lo que estás haciendo y a tomar tus propias decisiones. Creo que la mejor manera de aprender es validando tus propias acciones y eso es justo lo que hicimos en esta clase, no solo escuchar las opiniones de alguien más sino las propias".

Miguel mencionó que esta clase le "ayudó mucho a comprender cómo es el diseñar en el siglo XXI, distintas maneras de llegar a cumplir con la innovación, y diseñar no a partir de especulaciones que se tengan de una manera personal, sino, sustentar mis ideas en una investigación real."

Sofía menciona que le "gustó mucho experimentar este tipo de trabajo desde la perspectiva de facilitador porque te da un punto de vista distinto del trabajo de diseño, y puedes hacer observaciones objetivas sobre el proceso creativo que llevamos al diseñar. Me habría gustado que en mi sesión participaran personas que no fueran diseñadoras, porque creo que eso habría generado discusiones y propuestas en distintos campos y ámbitos (...)".

\section{Seis estrategias para el diseño de proyectos participativos}

En base a los aprendizajes obtenidos durante estos últimos años, he identificado seis estrategias resultado de la investigación y la experimentación con diferentes iniciativas educativas (sean estas propias o ajenas) que pretenden ofrecer un panorama moderno para pensar, crear e implementar nuevas prácticas participativas que promuevan la reflexión creativa de los participantes y la construcción de nuevas realidades más equitativas.

Estas estrategias me han permitido estructurar un marco teórico y metodológico a través del cual diseñar los planes de estudio de nuevos proyectos formativos en búsqueda de la generar ambientes participativos, propicios a la colaboración y a la creación colectiva. 


\section{De la educación del diseño al diseño de la educación}

Como diseñadores, conocemos la importancia de la investigación, sabemos que debemos primero identificar las necesidades del público para el que diseñamos y prestar especial atención en el contexto donde se llevará a cabo la interacción del usuario con nuestro diseño. Como educadores debemos procurar lo mismo: diseñamos nuestros cursos y es así como los deseos, las necesidades y el contexto de nuestros alumnos, deben estar presentes en todo momento.

Para aprender de nuestros estudiantes, debemos primero asumir el espacio educativo como uno de intercambio de experiencias y conocimientos, en donde todos tienen algo importante que aportar a la clase: el estudiante, por un lado, aprende del profesor los conocimientos y las experiencias que han marcado su vida y promueven su desarrollo personal y profesional. El profesor, a cambio, mantiene un asiento en primera fila que le permite observar el comportamiento de sus estudiantes, analizar el resultado de las técnicas que aplica, medir la asimilación de la teoría que imparte y mejorar, de manera continua, su labor como docente.

El carácter cíclico del sistema escolar se acomoda a la perfección para repetir, año tras año, un mismo curso, sin embargo, este nunca debe ser igual. Los estudiantes cambian y con ellos cambian también sus necesidades y su contexto. Los planes de estudio deben ser flexibles y permeables para integrar nuevas prácticas y conocimientos, que promuevan una experiencia educativa relevante y siempre fresca.

Con cada final del ciclo educativo, se presenta la oportunidad para validar el desempeño de la clase, un prototipo en constante desarrollo. Así pues, la evaluación del curso deberá contrastar los objetivos sentados al principio del curso con los resultados obtenidos tanto por los alumnos como por el docente.

El registro y la sistematización de datos durante y al final del curso nos brindan una cantidad extraordinaria de información. Mientras sepamos identificar y refinar las mejores prácticas desarrolladas durante las clases, podremos entonces mejorar la experiencia de los alumnos.

De compartirse los objetivos, el método y los resultados, nos permiten darnos a conocer y eventualmente abren la posibilidad de replicar los éxitos obtenidos en otros contextos, trabajando con poblaciones diferentes o bajo circunstancias alternas, que no teníamos contempladas. 


\section{Del producto creado al proceso creativo}

El objetivo del proceso de diseño ha dejado ya de ser el producto creado y cada vez se valora más el proceso creativo mediante el cual, se identifican y satisfacen las necesidades de los usuarios. Como menciona Jan Stappers (2008) cuando habla acerca de los nuevos paisajes del diseño: "ya no sólo diseñamos productos para usuarios. Diseñamos las experiencias futuras de la gente, las comunidades y las culturas, mismas que se encuentran actualmente interconectadas e informadas de maneras que habrían sido imposibles de imaginar hace apenas 10 años".

Mientras tradicionalmente se enfocaban los esfuerzos en diseñar productos, siendo estos objetos, edificios, gráficos o páginas de internet, actualmente se busca enriquecer el propósito que estos tienen en la vida de nuestros usuarios. Buscamos más allá del resultado tangible, es decir, diseñamos para hacer feliz a los usuarios, para promover comportamientos más sustentables o mejorar la experiencia de uso. Buscamos enriquecer las maneras en las que nos relacionamos entre seres humanos, y con el ambiente: buscamos contribuir al bienestar y a la felicidad de la gente que nos rodea.

Acorde a esta visión, enfocamos nuestra labor como docentes, no en un tema, sino en el diseño y la implementación de diversas herramientas (sean estas físicas, psíquicas o digitales) que permitan a nuestros estudiantes interactuar entre sí y expresar su creatividad sin contratiempos.

Este cambio, por pequeño que parezca, tiene incidencias cada vez más profundas en el quehacer del diseñador y del docente (Kalantzis \& Cope, 2010) y desata una serie de consideraciones a tomar en cuenta con respecto al foco de nuestra atención, sobre todo en el proceso de investigación previo a la conceptualización.

\section{Satisfaciendo necesidades de regeneración}

En su libro Desarrollo humano y ética para la sustentabilidad, Antonio Elizalde (2012) identifica tres tipos de sociedades en las que se conjugan, de distintas maneras, los comportamientos mediante los cuales la gente satisface sus necesidades. Menciona que hay sociedades consumistas donde las necesidades se satisfacen mediante la posesión de bienes. Existen otras, las sociedades ascéticas, donde los satisfactores y los bienes se 
derogan en búsqueda de la libertad espiritual. Finalmente, plantea una sociedad a la que denomina sustentable o ecológica

(...) donde lo que se trabaje preferentemente debe ser la oferta de satisfactores, tanto en calidad como en cantidad; de lo que se trata es de enriquecer las formas como damos cuenta de las necesidades humanas. Aquí es importante señalar lo siguiente: los satisfactores en cuanto son los elementos inmateriales de una cultura no tienen peso material, no generan una carga sobre el medio ambiente. Los satisfactores son las formas culturales, son lo más propiamente humano porque es lo que creamos culturalmente (Elizalde, 2010).

Servicios como Wikipedia, BlahBlahCar y Couchsurfing nos permiten observar algunos ejemplos de proyectos que promueven nuevos patrones de conducta donde se valoran el satisfactor - conocimiento, transporte y hospedaje - por sobre los bienes - enciclopedia, coche y albergue. Estas plataformas se erigen sobre los principios de colaboración al mismo tiempo que nos permiten hacer un uso más eficiente de los recursos.

Servicios de esta naturaleza suelen empezar desde abajo, contando con el soporte de pocos, pero con toda su creatividad e intención y son sólo ejemplos de los tantos proyectos regenerativos que se están sumado a la labor de construir nuevos satisfactores que resuelvan necesidades, inspirados por la gente que nos rodea y motivados por los sueños a los que aspiramos.

Mientras logremos integrar valores tales como la confianza, el respeto y la pasión en todos nuestros emprendimientos, podremos construir un mundo más equitativo, igualitario e incluyente.

\section{La industria a escala humana}

En este mismo tenor, me parece ya obsoleto el apellido "industrial" que seguimos poniéndole a la disciplina que nos formó a muchos de nosotros y con ello no quiero decir que haya que cambiarlo (podríamos simplemente tener a bien omitirlo), pero sí creo que la virtud de nuestro quehacer no se limita al tiraje, sino a nuestra capacidad para identificar y satisfacer las necesidades de la gente para la que trabajamos. 
Daniel Romero, profesor del CIDI y socio fundador de TUUX dice que "la producción a gran escala ya no es vista como el único trabajo verdadero. Anteriormente entre más piezas se fabricaban, mejor. En este esquema el éxito era medido como una ganancia monetaria. Nunca cerca de los pequeños talleres y los oficios tradicionales."

Asumir el desarrollo de nuevos productos dentro de un esquema local o regional involucra cambiar no solo los métodos productivos, sino prestar mayor atención a las necesidades de una población específica, a los recursos con los que se cuenta al interior de ésta y sobre todo a las relaciones humanas que se desarrollan alrededor de nuestro proyecto.

El enfoque en un usuario social da pie a un diseño centrado en la comunidad, en donde el individuo sigue siendo el medio de contacto, pero las necesidades que se busca satisfacer son las de un grupo, y es tomando en cuenta las relaciones entre los sujetos como podemos generar un impacto positivo en la vida de más personas, quienes al final son las poseedoras de esas maravillosas sonrisas que iluminan rostro y corazón.

\section{Trabajo y creatividad colectiva}

Seineldin (2011) compara la dinámica dentro de cualquier sociedad - la organización de las diferentes instancias que la conforman - con el funcionamiento del cuerpo humano, basado en el ejercicio interdependiente de los diferentes órganos que lo integran.

Siendo que el tejido social nunca antes había estado tan explícitamente interconectado como lo está ahora, la colaboración se vuelve fundamental, más aún cuando nos percatamos de que, si queremos enfrentar los retos a los que nuestra sociedad se está enfrentando, necesitamos trabajar en equipo.

Como dice David de Ugarte (2015).

Si el conocimiento es un hecho comunitario, y lo es, no tiene sentido pedir a ningún ente externo que haga las cosas que queremos o nos provea de aquello que necesitamos, porque nos estaríamos privando de la experiencia de hacerlas, lo que 
desde el punto de vista del conocimiento es tan importante muchas veces como la cosa en sí. Libertad es la posibilidad de hacerlas por nosotros mismos y si tiene sentido reclamar algo es que sean retiradas las trabas de cualquier tipo que nos impiden construir comunitariamente las herramientas del cambio Ugarte (2015).

En términos estadísticos, una comunidad diversa, independiente, descentralizada y coordinada tiende a ofrecer resultados más representativos del universo de posibles resultados (Surowiecki, 2004); en términos educativos, la vinculación entre distintas empresas profesionales y la coordinación con sus elementos sitúa al educando dentro de escenarios más parecidos a los que se encontrará en sus primeros años como profesionista y facilitando el tránsito de la vida estudiantil a la vida profesional.

La colaboración permite que todos los jugadores ganen y puede ser hacia adentro de la universidad, trabajando en conjunto con diferentes institutos y facultades, o hacia afuera, a través de instancias externas: sociales, públicas o privadas. De ambas maneras, se permite a los estudiantes desenvolverse en espacios multidisciplinarios, en donde el trabajo en equipo es fundamental para la satisfacción de los objetivos identificados y al mismo tiempo les permite a las entidades participantes la oportunidad de aprender de los estudiantes y de integrar nuevas perspectivas en su quehacer productivo.

\section{Artefactos para propiciar el diálogo}

En el libro Convivial Toolbox, Sanders y Stappers (2012) explican el cómo y el porqué del uso de herramientas generativas, aquellas que buscan activar a la gente a hacer y a reflexionar sobre lo hecho. Los autores señalan que estas herramientas pueden ofrecer a los participantes en una sesión exploratoria un mejor acceso a su conocimiento latente, mismo que podría quedar en el ámbito inconsciente o pasar desapercibido en los métodos tradicionales de investigación.

Brandt (2006), Boess, et at (2011) y Ten Bhömer, et al (2013) son algunos de los investigadores que se han dado a la tarea de documentar el efecto que ciertos prototipos interactivos, juegos y otros artefactos pueden tener en el devenir de sesiones participativas, donde los diferentes integrantes colaboran en la exploración, elaboración y confrontación de diferentes ideas y conceptos de diseño dentro de un ambiente co-creativo. 
La dirección de sus investigaciones indica que el uso de juegos y prototipos interactivos incita al diálogo y al intercambio de conocimiento y experiencias, promueve la colaboración y permiten a los participantes tomar decisiones complejas, tomando en cuenta los múltiples aspectos que en cada caso merecen ser considerados. El diseño de estos artefactos integra el punto de vista de diferentes participantes quienes suelen convertirse en los primeros usuarios y promotores. A través de múltiples iteraciones, los involucrados en el proyecto pueden refinar las pautas interactivas y ampliar el impacto de sus emprendimientos. Los juegos y las dinámicas lúdicas para sensibilizar a los participantes y motivar su participación libre de inhibición pueden, por momentos, parecer absurdos o fuera de lugar; sin embargo, la retroalimentación por parte de estudiantes y representantes de las empresas nos brinda evidencia para validar su importancia.

\section{Conclusiones}

La presente materia comenzó buscando combinar las habilidades de los alumnos con las necesidades de los emprendedores y viceversa; es así como, después de tres iteraciones, he logrado identificar ciertas actitudes y actividades que facilitan la vinculación y promueven la colaboración entre más de 50 alumnos que han tomado la clase y 24 jóvenes emprendedores.

Los resultados aquí expuestos son solo un paso más en la creación de espacios que promuevan la cultura del diálogo y la participación ciudadana, procurando la vinculación y el cambio social desde una perspectiva transdisciplinaria en donde múltiples jugadores colaboran en proyectos personales y colectivos. La labor no acaba aquí y es así que estoy actualmente por terminar una siguiente iteración del curso, habiendo cambiado algunas cosas y otras no. El objetivo original sigue siendo el mismo, pero con una carga social más evidente. En vez de proveer a los alumnos con el contacto de una empresa preseleccionada, este semestre les di la responsabilidad a los alumnos de definir la naturaleza de los emprendimientos a los que se vincularían durante el semestre. La toma de decisiones ha sido desde el principio a través de la interacción con vecinos y transeúntes en el espacio público y es así que al día de hoy, los alumnos están trabajando en sus segundos prototipos y prontos a presentar los resultados del proceso de participativo ante la comunidad de un parque. 


\section{Limitaciones}

El curso de CoDiseño se centra en el diseño y la puesta en práctica de técnicas y herramientas para impulsar un proceso creativo plural y organizado. La evaluación del alumno se basa en la puntualidad, la participación, la integración de los principios de facilitación creativa, el manejo de sesiones participativas, la documentación de los procesos y la reflexión con respecto a su participación en las dinámicas grupales; teniendo un carácter predominantemente académico, la producción y la comercialización de las propuestas diseñadas, queda fuera del alcance del curso. 
CODISEÑO Y TECNOLOGÍAS PARTICIPATIVAS

\section{Agradecimientos}

Agradezco a todas las personas e instituciones que han participado en este proyecto y nos han permitido levantar la voz para generar consciencia a cerca de la importancia de trabajar en equipo, de promover el entendimiento del otro y la creación colectiva de nuevas y mejores realidades. Gracias a Héctor López Aguado por invitarme a dar clases al CIDI por primera vez, a Laura Sánchez, Karina Cocho, Rosa María Ledesma y Enriqueta Tapia, personal administrativo de la Facultad que me han brindado su ayuda en todo momento para lidiar con los asuntos complementarios a la clase; a Gabriel Calvillo, Aleida Mota, Iván Pérez Toledo, Diego Rodríguez, Lisel Enríquez, Marina Acevedo, Sofía Vega, Laura Vega, Ariana Méndez, Leonardo Pérez y Pérez, Juan Vértiz, Eva Zamarripa y Marlen Carmona, estudiantes de la primera generación que tomaron el curso de CoDiseño sin saber lo que les esperaba y me dieron su confianza para comenzar con este afortunado experimento; a Juan del Cerro de BonKrep; Óscar Velázquez y Anaí Aguilar del Creatorspace, Karina Caudillo e Iván Palomera de Smartup, Cecilia Mojica de Delinutrición, Ana Lucía Coll y María Huerta de lluméxico, Christian Tapia de Mutante, Margarita González y José Luis Muñiz de New Ventures, Paulina Moreno, Begoña Ortíz y Cristina Guash de Las Páginas Verdes y Carlos Apipilhuasco, Nelly Mejía y Jahir Mojica de SUEMA, representantes de las empresas con las que colaboramos durante este primer semestre; a Alan Hazel Godoy, Alejandro Meza, Andrea Quintana, Arantxa Calderón, Diego Velázquez, Ariel Valle, lliana Ordoñez, Imelda Moreno, Iraís Medina, Jorge Molina, Karen Guerrero, Rocío Calderón, Rodrigo Guerrero, Roxana López y Sebastián Nino, alumnos de la segunda generación; a Alejandro Fernández y Akemi Sato de m+ubano, Eluzai Perdomo de Inven3, Alejandra Hernández de Natupla, Marco Butrón, Cesar González y Fabián Alfonso de Triquitraqueando, Alicia Barceinas, de Natura Mexicana, Valeria Lemus del Foro Shakespeare, Priscila Alanis de la Universidad del Medio Ambiente, Elias Cattán de Taller13 y Karla Arana, Armando Álvarez, Javier Fajardo y Noé Castillo de SUEMA, representantes de las empresas con las que colaboramos durante el segundo semestre de la edición de CoDiseño; a Andrea Naranjo, Arturo Flores, Daniel Linares, Daniela Suchil, Donaldo Preciado, Georgina Reza, Lizbeth Ortiz, Michelle Calderón, Miguel Angel Lobato, Nancy Valerdi, Pablo García, Paola González, Paola Palomino y Rafael Gutiérrez, alumnos de la tercera generación de la materia; a Tania Ortíz, Liz Sánchez, Carlos Zimbrón y Luis D’Nava de WeAreTodos, Leticia Gasca y Yannick Kwik de FuckUpNights, representantes de las empresas con las que colaboramos durante el tercer semestre; a Alejandra Núñez, Analy Miranda, Andrés Velázquez, Carolina Vieyra, Daniela Pérez, Desire Barrientos, Diana Nápoles, Diego Dualo, Fabiola López, Mariana Alanis, Paola Martínez, Sergio Hernández y Uriel Espinoza, estudiantes de la cuarta generación que se encuentran cursando actualmente la materia. Finalmente, quiero agradecer a mis padres, Gabriela Guzmán y Gerardo Alatorre y a mi hermano, Darío Alatorre, por compartir tan largas jornadas de discusión dentro de las cuales hemos sentado las bases que inspiran y estructuran el presente proyecto. 


\section{Referencias}

Acaraglou, L. (2014). Make change, New Your, US: Disrupt Design LLC

Brandt, E. (2006). Designing Exploratory Design

Games: A Framework for Participation in Participatory Design? [Participatory Design Conference]. Trento,Italia.

Boess, S., Pasman, G. \& Mulder, I. (2011). Making for Participation, [Participatory Innovation Conference]. Sønderborg, Dinamarca.

Elizalde Hevia, A. (2012). Desarrollo Humano Sustentable: sus exigencias éticas, económicas y políticas. Recuperado de: uv.mx/mie/files/2012/10/ SESION-9-Elizande-Exigencias-Eticas-Socials-DS.pdf

Kalantzis, M. \& Cope, B. (2010), The Teacher as Designer: pedagogy in the new media age, E-Learning and Digital Media Vol. 7 No. 3

Klimenko, O. (2008). La creatividad como un desafí para la educación del siglo XXI, Educación y Educadores, Vol. 11, No. 2. Recuperado de redalyc.org/pdf/834/83411213.pdf

Manson, P. (2015). The end of capitalism has begun, The Guardian. Recuperado de theguardian.com/ books/2015/jul/17/ postcapitalism-end-of-capitalism-begun

Maturana, H. (1997). Metadesign. Recuperado de: inteco.cl/articulos/006/texto_ing.htm
Sanders, E. \& Stappers, P. J. (2008). Co-creation and the new landscapes of design. Recuperado de: maketools.com/articles-papers/CoCreation_ Sanders_Stappers_08_preprint.pdf

(2012). Convivial Toolbox, The Netherlands: BIS Publishers

Schlierf, K.; Boni Aristizábal, A. \& Lozano Aguilar J. F. (2008). La transferencia de tecnología participativa desde la universidad. Una oportunidad para la formación de una ciudadanía crítica, Departamento de Proyectos de Ingeniería, Universidad Politécnica de Valencia.

Seineldin, F. (2011). Experiencia Endeavor, Talk, Recuperado de youtube.com/watch?v=5hsO03YN9v4

Surowiecki, James. (2004). The Wisdom of the Crowds, Anchor Books.

Tassoul, Marc. ( 2009). Creative Facilitation, 3ra ed, VSSD, Netherlands.

Ten Bhömer, M. Brouwer, C.;Tomico, O. \& Wensveen, S. (2013). Interactive prototypes in the participatory development of product-service systems, Participatory Innovation Conference. Lahti, Finland.

Ugarte, D. (2015). Ética y política de la abundancia, El correo de las indias. Recuperado de: lasindias. com/etica-y-politica-de-la-abundancia. 